6. P. R. Halmos, The range of a vector measure, Bull. Amer. Math. Soc. vol. 54 (1948) pp. $416-421$.

7. R. R. Phelps, Uniqueness of Hahn-Banach extensions and unique best approximation, Trans. Amer. Math. Soc. vol. 95 (1960) pp. 235-255.

Massachusetts Institute of Technology

\title{
THE GEÖCZE $k$-AREA AND A CYLINDRICAL PROPERTY
}

\section{TOGO NISHIURA}

In [5], a definition of the Geöcze $k$-area of a mapping from admissible sets of Euclidean $k$-space $E_{k}$ into Euclidean $n$-space $E_{n}(2 \leqq k \leqq n)$ is given. This definition is an extension of the Geöcze area given in [3]. With this definition of Geöcze $k$-area, a treatment of Geöcze $k$-area is developed for flat mappings $(k=n)$ paralleling the treatment of Geöcze area for plane mappings given in [3]. The present paper gives results concerning the Geöcze $k$-area for mappings from admissible sets of $E_{k}$ into $E_{n}(n>k \geqq 2)$. A cylindrical property is defined for mappings in harmony with $[3,(16.10)]$. This property, which has had an essential part in the proofs of the main theorems for Lebesgue area for mappings from admissible sets of $E_{2}$ into $E_{n}$ [3] and which has been used in other research, is shown to play a prominent role in the extension of the theory of Geöcze area to higher dimensions. An example is given to show that the theorems concerning the cylindrical property in [3] are no longer valid for $k \geqq 3$. These theorems are shown to be valid under a certain restrictive hypothesis found in the literature.

1. Notations and definitions. If $X$ is a set in $E_{k}$, then $\bar{X}, X^{0}$, and $X^{*}$ will denote respectively the closure, interior, and boundary of $X$.

A polyhedral region $R$ in $E_{k}$ is the point-set covered by a strongly connected $k$-complex situated in $E_{k}$. A polyhedral region $R$ is called simple if $E_{k}-R$ is connected (see [5]).

By a figure $F$ we mean a finite union of nonoverlapping polyhedral regions in $E_{k}$ such that the interior of the union is the union of the interior of the finitely many polyhedral regions. A set $A$ in $E_{k}$ is said to be admissible in each of the following cases:

Presented to the Society, January 29, 1960 under the title The Geöcze k-area; received by the editors September 21, 1960. 
(a) $A$ is an open set in $E_{k}$.

(b) $A$ is a figure.

(c) $A$ is a homeomorphic image of a figure.

(d) $A$ is a set open in the type (b) or (c) above.

By $\Im(k, n)$ we mean the set of all continuous mappings $(T, A)$ from admissible sets $A$ in $E_{k}$ into $E_{n}(2 \leqq k \leqq n)$. A continuous mapping $(T, A)$ is called fat if $(T, A) \in J(k, k)$.

Let $\left(T_{0}, A_{0}\right),\left(T_{j}, A_{j}\right)(j=1,2, \cdots)$ be a sequence of mappings in $\Im(k, n) .\left(T_{j}, A_{j}\right)(j=1,2, \cdots)$ is said to converge to $\left(T_{0}, A_{0}\right)$ if

(i) $A_{j} \subset A_{j+1} \subset A_{0}$ for all $j$;

(ii) $\bigcup_{j=1}^{\infty} A_{j}^{0}=A_{0}^{0}$;

(iii) Sup $\left[\left|T(w)-T_{j}(w)\right|: w \in A_{j}\right] \rightarrow 0$ as $j \rightarrow \infty$.

If $k<n$ are positive integers then $\Omega_{n}^{k}$ is the set of all $k$-termed sequences $\xi=\left(\xi_{1}, \xi_{2}, \cdots, \xi_{k}\right)$ of integers such that $1 \leqq \xi_{1}<\xi_{2}<\cdots$ $<\xi_{k} \leqq n$. We shall assume that $\Omega_{n}^{k}$ is lexicographically ordered.

If $x=\left(x^{1}, x^{2}, \cdots, x^{n}\right) \in E_{n}$ then by the mapping $P_{n}^{\xi}, \xi \in \Omega_{n}^{k}$, we shall mean the projection of $E_{n}$ onto the coordinate hyperspace $E_{k}^{\xi}$ where $P_{n}^{\xi}(x)=\left(x^{\xi_{1}}, x^{\xi_{2}}, \cdots, x^{\xi k}\right) \in E_{k}^{\xi}$.

If $(T, A) \in J(k, n)$ then by $\left(T^{\xi}, A\right)$ we mean the mapping $\left(P_{n}^{\xi} T, A\right)$, $\xi \in \Omega_{n}^{m}, k \leqq m \leqq n$. If $m=k$ then $(T \xi, A), \xi \in \Omega_{n}^{k}$, forms a collection of $C_{k}^{n}$ flat mappings from the admissible set $A$ into $E_{k}^{\xi} \subset E_{n}, \xi \in \Omega_{n}^{k}$.

Let $(T, A) \in J(k, n)$ and $D$ be any bounded open connected set such that $\bar{D} \subset A$. If $C^{\xi}:\left(T^{\xi}, D^{*}\right), \xi \in \Omega_{n}^{k}$, then a topological index $O\left(x ; C^{\xi}\right), x \in E_{k}^{\xi}$, is defined $\{$ see $[5,(1.3)]$ and also $[6 ;$ p. 125] $\}$. The function $O\left(x ; C^{\xi}\right)$ is a measurable function of $x \in E_{\mathbf{k}}^{\xi}$ and the integral $v\left(T^{\xi}, D\right)=\left(E_{k}^{\xi}\right) \int\left|O\left(x ; C^{\xi}\right)\right|$ exists (finite or infinite) for each $\xi \in \Omega_{n}^{k}$. $\left[v\left(T^{\xi}, D\right): \xi \in \Omega_{n}^{k}\right]$ forms a $1 \times C_{k}^{n}$ vector. Denote by $v(T, D)$ the Euclidean norm of this vector; i.e., $v(T, D)=\left[\sum v^{2}\left(T^{\xi}, D\right)\right]^{1 / 2}$, where $\sum$ ranges over $\xi \in \Omega_{n}^{k}$. Denote by $\mathfrak{S}$ a finite system of nonoverlapping simple polyhedral regions $\pi \subset A$. We define the Geöcze k-area of $(T, A)$ to be the number $V(T, A)=\operatorname{Sup} \sum v(T, \pi)$, where $v(T, \pi)$ $=v\left(T, \pi^{0}\right), \sum$ ranges over $\pi \in \subseteq$ and the supremum is taken over all possible systems $\subseteq$. If $V(T, A)<\infty$, then the integral $u\left(T^{\xi}, D\right)$ $=\left(E_{\mathbf{k}}^{\xi}\right) \int O\left(x ; C^{\xi}\right)$ exists for each $\xi \in \Omega_{n}^{k}$. Let $u(T, D)=\left[\sum u^{2}\left(T^{\xi}, D\right)\right]^{1 / 2}$, and $U(T, A)=\operatorname{Sup} \sum u(T, \pi)$, where $u(T, \pi)=u\left(T, \pi^{0}\right)$ and the sum $\sum$ and the supremum are taken as above.

Let $(T, A) \in J(k, n)$. Then $\Gamma(T, A)$ will denote the collection of all maximal connected sets of $A$ on which $(T, A)$ is constant. If $A$ is compact then $\Gamma(T, A)$ forms an upper semicontinuous decomposition of $A$.

2. The cylindrical property. In this section we confine ourselves to continuous mappings $(T, A) \in J(k, n)$ with $A$ compact and $n>k$. For 
such mappings we have $C_{\boldsymbol{k}}^{\boldsymbol{n}}$ flat mappings $\left(T^{\xi}, A\right), \xi \in \Omega_{n}^{\boldsymbol{k}}$. Associated with these mappings are their corresponding upper semicontinuous decompositions $\Gamma(T, A), \Gamma\left(T^{\xi}, A\right), \xi \in \Omega_{n}^{\boldsymbol{k}}$, of $A$. A point $x \in E_{\boldsymbol{k}}^{\xi}$ is said to have the cylindrical property with respect to $(T, A)$ if (i) $x \in T^{\xi}(A)$; (ii) there is a $g \in \Gamma\left(T^{\xi}, A\right)$ such that $T^{\xi}(g)=x$ and $T(g)$ is nondegenerate.

2.1. Theorems concerning the cylindrical property. For each $\xi \in \Omega_{n}^{k}$ let $X^{\xi}$ be the set of all $x \in E_{\mathbf{k}}^{\xi}$ having the cylindrical property with respect to $(T, A)$.

Theorem (i). Let $(T, A) \in J(2, n)$ with $A$ compact and $V(T, A)<\infty$. Then, for each $\xi \in \Omega_{n}^{2}, X^{\xi}$ has zero Lebesgue 2-measure.

Proof. This theorem was first proved by L. Cesari in [2] for the case $n=3$. It is easily shown that this special case implies the theorem. Another proof is also given in [4, Theorem 8.12].

Theorem (i) above shows that for $k=2$, the condition $V(T, A)$ is finite has very strong implications, namely, the set $X^{\xi}$ has zero measure for all $\xi \in \Omega_{n}^{2}$. This no longer is true when $k>2$ as an example in (2.2) below shows. The following theorem gives a sufficient condition on the mapping $(T, A) \in J(k, n)$ to make the Lebesgue $k$-measure of $X^{\xi}$ zero, $\xi \in \Omega_{n}^{k}, n>k \geqq 2$.

Theorem (ii). Let $(T, A) \in J(k, n)(2 \leqq k<n), A$ be compact and $H_{n}^{k+1}[T(A)]=0$, where $H_{n}^{k+1}[\cdot]$ denotes the $(k+1)$-dimensional Hausdorff measure in $E_{n}$. Then $m_{k}\left[X^{\xi}\right]=0$ for all $\xi \in \Omega_{n}^{k}$, where $m_{k}[\cdot]$ is the $k$-dimensional Lebesgue measure.

Proof. Let $\alpha \in \Omega_{n}^{k+1}, P_{n}^{\alpha}$ and $E_{k+1}^{\alpha}$ be defined as in $\$ 1$ above. Let us fix a $\xi \in \Omega_{n}^{k}$. For each $\alpha \in \Omega_{n}^{k+1}$ with $E_{k}^{\xi} \subset E_{k+1}^{\alpha}$, let $X_{\alpha}^{\xi}$ denote the set of all points of $E_{k}^{\xi}$ which have the cylindrical property with respect to the mapping $\left(P_{n}^{\alpha} T, A\right)$. Then we have that $X^{\xi}=U X_{\alpha}^{\xi}$, where $U$ ranges over all $\alpha \in \Omega_{n}^{k+1}$ with $E_{k}^{\xi} \subset E_{k+1}^{\alpha}$. Hence it is sufficient to show that $m_{k}\left[X_{\alpha}^{\xi}\right]=0$ for each $\alpha \in \Omega_{n}^{k+1}$ with $E_{k}^{\xi} \subset E_{k+1}^{\alpha}$.

Since $0=H_{n}^{k+1}[T(A)] \geqq H_{k+1}^{k+1}\left[P_{n}^{\alpha} T(A)\right]=m_{k+1}\left[P_{n}^{\alpha} T(A)\right] \geqq 0, \alpha \in \Omega_{n}^{k+1}$, we have $m_{k+1}\left[P_{n}^{\alpha} T(A)\right]=0$ for each $\alpha \in \Omega_{n}^{k+1}$. This implies that we need only study the case where $(T, A)$ is a continuous mapping of the compact admissible set $A \subset E_{k}$ into $E_{k+1}$ with $m_{k+1}[T(A)]=0$. Suppose this is the case and let $\xi \in \Omega_{k+1}^{k}$. For the sake of simplicity, let $\xi$ be such that $E_{k}^{\xi}=\left\{x \in E_{k+1}: x^{1}=0\right\}$. Let $\left[a_{i}, b_{i}\right], i=1,2, \cdots$, be the countable collection of closed intervals on the $x^{1}$-axis of $E_{k+1}$, where $a_{i}$ and $b_{i}$ are rational numbers, $a_{i}<b_{i}$. Denote by $P$ the usual projection of $E_{k+1}$ onto the $x^{1}$-axis. If $X_{i}^{\xi}$ denotes the set of all $x \in X^{\xi}$ with the property that $\left(T^{\xi}\right)^{-1}(x)$ has a component $g$ such that $P T(g)$ 
covers $\left[a_{i}, b_{i}\right]$, then $X^{\xi}=\bigcup_{i=1}^{\infty} X_{i}^{\xi}$. Since $A$ is compact and $\Gamma\left(T^{\xi}, A\right)$ is an upper semicontinuous decomposition of $A, X_{i}^{\xi}$ is closed and hence compact for each $i$.

Let $Z_{i}=T(A) \cap\left(P_{\mathbf{k}_{+1}}^{\xi}\right)^{-1}\left(X_{i}^{\xi}\right) . Z_{i}$ is compact and hence measurable. Hence for each $i, 0=m_{k+1}[T(A)] \geqq m_{k+1}\left[Z_{i}\right] \geqq m_{k}\left[X_{i}^{\xi}\right] \cdot\left(b_{i}-a_{i}\right) \geqq 0$. Therefore $m_{k}\left[X_{i}^{\xi}\right]=0$. Thus we conclude that $m_{k}\left[X^{\xi}\right]=0$ and the theorem is proved.

2.2. EXAMPLE. We give an example to show that in (2.1.ii) above, the condition $H_{n}^{k+1}[T(A)]=0$ cannot be replaced by $V(T, A)<\infty$ if $n>k=3$. This example will be used again in the next section.

Let $A=\left\{w \in E_{3}: 0 \leqq w^{i} \leqq 1, i=1,2,3\right\}, A_{0}=\left\{w \in A: w^{3}=0\right\}$, and let $P_{0}$ be the usual projection map of $A$ onto $A_{0}$. To facilitate discussion, we will denote the $m$-dimensional hyperspace of $E_{n}$ spanned by $\left(x^{1}, x^{2}, \cdots, x^{m}, 0, \cdots, 0\right)$ by $E\left(x^{1}, x^{2}, \cdots, x^{m}\right)$.

Let $\left(T_{0}, A_{0}\right): x^{i}=f^{i}\left(w^{1}, w^{2}\right), w \in A_{0}, i=1,2,3$, be the continuous mapping defined by A. Besicovitch in [1] of the square $A_{0}$ into $E\left(x^{1}, x^{2}, x^{3}\right)$ so that $\left(T_{0}, A_{0}\right)$ is a homeomorphism, $m_{3}\left[T_{0}\left(A_{0}\right)\right]>0$ and the 2 -area of $\left(T_{0}, A_{0}\right)$ is a positive number $\epsilon$. Let $(T, A)$ be defined by $x^{i}=f^{i}\left(w^{1}, w^{2}\right), i=1,2,3, x^{4}=w^{3}, w=\left(w^{1}, w^{2}, w^{3}\right) \in A$. Clearly $(T, A)$ is a homeomorphism. Hence every element of $\Gamma(T, A)$ is a point $w \in A$. Let $\xi \in \Omega_{4}^{3}$ be such that $E_{3}^{\xi}=E\left(x^{1}, x^{2}, x^{3}\right)$. Then every element $g \in \Gamma\left(T^{\xi}, A\right)$ is an interval. Hence $X^{\xi}=T^{\xi}(A)$, and $m_{3}\left[X^{\xi}\right]$ $=m_{3}\left[T^{\xi}(A)\right]=m_{3}\left[T_{0}\left(A_{0}\right)\right]>0$, since $T^{\xi}(w)=T_{0} P_{0}(w)$.

Let us show $V(T, A) \leqq \epsilon$. Since the 2 -area of $\left(T_{0}, A_{0}\right)$ is $\epsilon$, by $[3,(24.1 . i)]$, there exists a sequence of quasi-linear mappings $\left(Q_{0 j}, A_{0}\right): x^{i}=h_{j}^{i}\left(w^{1}, w^{2}\right), i=1,2,3, w \in A_{0}, j=1,2, \cdots$, converging to $\left(T_{0}, A_{0}\right)$ and the 2-area of $\left(Q_{0 j}, A_{0}\right)$ converging to $\epsilon$. Let $\left(Q_{j}, A\right)$ be the quasi-linear mapping $x^{i}=h_{j}^{i}\left(w^{1}, w^{2}\right), i=1,2,3, x^{4}=w^{3}, w \in A$, $j=1,2, \cdots$. Clearly, $\left(Q_{j}, A\right) \rightarrow(T, A)$ as $j \rightarrow \infty$. By [5, (2.2.x)], $V\left(Q_{j}, A\right)$ is equal to the 2 -area of $\left(Q_{0 j}, A_{0}\right)$. Hence by the lower semicontinuity of the Geöcze $k$-area, $\epsilon=\lim _{j \rightarrow \infty} V\left(Q_{j}, A\right) \geqq V(T, A)$ $[5,(2.2 . v i)]$. Thus the example is constructed.

3. The indices $d, m, \sigma$. In the theory of Geöcze area, the indices $d, m, \sigma$ play an important role (see [3]). These indices have been defined for the Geöcze $k$-area $[5,(3.3)]$. We give these definitions below.

Let $(T, F) \in \Im(k, n)$ where $F$ is a figure. Denote by $\mathfrak{S}=\mathbb{S}^{\prime} \cup \mathbb{S}^{\prime \prime}$ a finite subdivision of $F$ into nonoverlapping polyhedral regions, where $\widetilde{S}^{\prime}$ is the collection of simple polyhedral regions and $\widetilde{S}^{\prime \prime}$ is the collection of nonsimple polyhedral regions. Then $d=\max [\operatorname{diam} T(R): R \in \mathfrak{S}$ ]. $m=\max \left[m_{k}\left[\cup T^{\xi}\left(R^{*}\right)\right]: \xi \in \Omega_{n}^{k}\right]$, where $U$ ranges over all $R \in \mathbb{S}^{\prime}$ with $R \subset F^{0}, \sigma=\max \left[\sigma^{\xi}: \xi \in \Omega_{n}^{k}\right]$, where $\sigma^{\xi}=\sum^{\prime \prime} \sum^{*} \operatorname{diam} T^{\xi}\left(B_{i}\right)$, with $\sum^{*}$ 
ranging over all components $B_{i}$ of $R^{*}$ and $\sum^{\prime \prime}$ ranging over all $R \in \mathfrak{S}^{\prime \prime}, R \subset F^{0}$.

Theorem (i). If $k=2$ and $V(T, F)<\infty$, then for every $\epsilon>0$ there is a subdivision $\mathfrak{S}=\mathfrak{S}^{\prime} \cup \mathfrak{S}^{\prime \prime}$ of $F$ with indices $d, m, \sigma<\epsilon$.

THEOREM (ii). If $k \geqq 2$ and $H_{n}^{k+1}[T(F)]=0$, then for every $\epsilon>0$ there is a subdivision $\mathfrak{S}=\mathfrak{S}^{\prime} \cup \mathfrak{S}^{\prime \prime}$ of $F$ with indices $d, m, \sigma<\epsilon$.

Proof. The proofs of Theorems (i) and (ii) are essentially the same as the proof of $[3,(21.1 . \mathrm{i})]$. The only change needed is the replacement of $[3,(16.10 . \mathrm{i})]$ by (2.1.i) and (2.1.ii), respectively.

We observe that in the example of (2.2), $d+m+\sigma>\epsilon_{0}>0$ for an appropriate $\epsilon_{0}$, and $H_{n}^{k+1}[T(F)] \neq 0$.

4. Theorems on limits with respect to $d, m, \sigma$. Let $(T, A) \in J(k, n)$, $F_{j}(j=1,2, \cdots)$ be a sequence of figures with $F_{j} \subset F_{j+1} \subset A$ and $\bigcup_{j=1}^{\infty} F_{j}^{0}=A^{0}$, and $\mathfrak{S}_{j}=\mathfrak{S}_{j}^{\prime} \cup \mathfrak{S}_{j}^{\prime \prime}$ be a subdivision of $F_{j}$ witn indices $d_{j}, m_{j}, \sigma_{j}(j=1,2, \cdots)$.

Theorem (i). If $A_{0} \subset A$ is an admissible set and $d_{j}+m_{j}+\sigma_{j} \rightarrow 0$ as $j \rightarrow \infty$, then

$\lim _{j \rightarrow \infty} \sum^{(i)} v(T, q)=V\left(T, A_{0}\right), \quad \lim _{j \rightarrow \infty} \sum^{(i)} v\left(T^{\xi}, q\right)=V\left(T^{\xi}, A_{0}\right), \quad \xi \in \Omega_{n}^{k}$,

where $\sum^{(j)}$ ranges over all $q \in \mathfrak{S}_{j}^{\prime}, q \subset A_{0}$.

If, furthermore, $V(T, A)<\infty$, then

$$
\begin{aligned}
\lim _{j \rightarrow \infty} \sum^{(i)} u(T, q) & =U\left(T, A_{0}\right)=V\left(T, A_{0}\right), \\
\lim _{j \rightarrow \infty} \sum^{(i)}\left|u\left(T^{\xi}, q\right)\right| & =U\left(T^{\xi}, A_{0}\right)=V\left(T^{\xi}, A_{0}\right), \quad \xi \in \Omega_{n}^{k},
\end{aligned}
$$

where $\sum{ }^{(j)}$ ranges over all $q \in \mathfrak{S}_{j}^{\prime}, q \subset A_{0}$.

Proof. The proof of (i) is essentially the same as [3, (21.2.i), (21.3.i, ii)].

From (3.i, ii) we have sufficient conditions on $(T, A)$ for the existence of subdivisions $\mathfrak{S}=\mathfrak{S}^{\prime} \cup \mathfrak{S}^{\prime \prime}$ of $F \subset A$ with arbitrary small indices $d, m, \sigma$. Namely, (1) $k=2$ and $V(T, A)<\infty$; or (2) $k \geqq 2$ and $H_{n}^{k+1}[T(A)]=0$.

5. In this section we give some theorems concerning the Geöcze $k$-area of mappings $(T, A) \in J(k, n)$.

TheOREM (i). If $(T, A) \in J(k, n)$ and $H_{n}^{k+1}[T(A)]=0$, then 
$\left[\sum V^{2}\left(T^{\xi}, A\right)\right]^{1 / 2} \leqq V(T, A) \leqq \sum V\left(T^{\xi}, A\right)$, where $\sum$ ranges over $\xi \in \Omega_{n}^{k}$.

Theorem (ii). Let $(T, A) \in J(k, n)$ with $H_{n}^{k+1}[T(A)]=0$ and $H \subset A$ be a set closed in $A$ such that $m_{k}\left[T^{\xi}\left(A^{0} \cap H\right)\right]=0, \xi \in \Omega_{n}^{k}$. Then each component $A_{i}$ of $A-H$ is an admissible set and $V(T, A)=\sum V\left(T, A_{i}\right)$, $V\left(T^{\xi}, A\right)=\sum V\left(T^{\xi}, A_{i}\right), \xi \in \Omega_{n}^{k}$, where $\sum$ ranges over all components $A_{i}$ of $A-H$.

TheOREM (iii). Let $(T, A) \in J(k, n)$ and $H_{n}^{k+1}[T(A)]=0$. Let $A_{i} \subset A(i=1,2, \cdots)$ be a collection of sets open in $A$ such that each $A_{i}$ is the union of sets $g \in \Gamma(T, A)$. Then we have $V\left(T, \cup A_{i}\right) \leqq \sum V\left(T, A_{i}\right)$ and $V\left(T^{\xi}, \cup A_{i}\right) \leqq \sum V\left(T^{\xi}, A_{i}\right), \xi \in \Omega_{n}^{k}$, where $U$ and $\sum$ range over all $A_{i}$.

The proofs of the above theorems are essentially the same as [3, (21.4.i), (21.5.i), (25.1.iii)].

\section{BIBLIOGRAPHY}

1. A. S. Besicovitch, Parametric surfaces, Bull. Amer. Math. Soc. vol. 56 (1950) pp. 288-296.

2. L. Cesari, Caratterizzazione analitica delle superficie continue di area finita secondo Lebesgue, Ann. Scuola Norm. Sup. Pisa vol. 10 (1941) pp. 253-294; vol. 11 (1942) pp. $1-42$.

3. - Surface area, Princeton University Press, 1956.

4. H. Federer, Measure and area, Bull. Amer. Math. Soc. vol. 58 (1952) pp. 306378.

5. T. Nishiura, The Geöcze $k$-area and flat mappings, to appear.

6. T. Rado and P. V. Reichelderfer, Continuous transformations in analysis, Berlin, Springer, 1955.

UNIVERSity OF Wisconsin, MiLWAUKeE 